\title{
Off-label utilization of antipsychotics
}

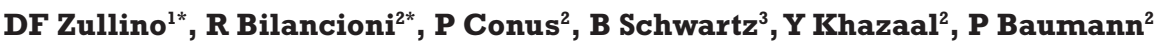 \\ *The contributions of these authors have to be considered as equivalent \\ 'Division of Substance Abuse, University Hospitals, Geneva, Switzerland \\ 2University Department of Adult Psychiatry, Prilly-Lausanne, Switzerland \\ ${ }^{3}$ Private practice, Geneva, Switzerland
}

\begin{abstract}
Objective: The newer atypical antipsychotics are prescribed because of their enhanced safety profiles and their larger pharmacological profile in comparison to the conventional antipsychotics. This has led to broad off-label utilisation. The aim of the present survey was to study the prescribing practice of hospital psychiatrists with regard to antipsychotic drugs, comparing patients treated for psychoses or other registered indications to patients receiving off-label antipsychotic treatment. Methods: As part of a pharmacovigilance/pharmacoepidemiology program, all drugs given on 5 reference days (1999 - 2001) in the 98bed psychiatric hospital of the University of Lausanne, Switzerland, were recorded along with age, sex, and diagnosis. The prescriptions of 202 patients were assessed. Patients were classified in 3 diagnostic groups: (1) patient with psychotic disorders, (2) patients with manic episodes and depressive episodes with psychotic symptoms, and (3) patients with other disorders. Group (1) and (2) formed the class of patients receiving an antipsychotic for a registered indication, and the prescriptions in group (3) were considered as off-label. Results: A lesser number of psychotic patients received antidepressant $(p<0.05)$ and nonbenzodiazepine hypnotics $(\mathrm{p}<0.001)$ compared to the patients of the other two groups. The patients with affective disorders seldom received a combination of an atypical and a conventional antipsychotic, whereas a lesser number of patients with offlabel indications received atypical antipsychotics less often than those of the two comparison groups ( $<<0.05)$. Stepwise logistic regression revealed that patients with a psychotic disorder were more likely to receive an antipsychotic medication in medium or high doses $(\mathrm{p}<0.001)$, in comparison to the two other groups. Conclusion: The new antipsychotic drugs seem to be prescribed with less hesitation and mainly for approved indications. Physicians prescribed new drugs, off-label, only after having gained some experience in the field of the approved indications, and were more cautious with regard to doses when treating on an offlabel basis.
\end{abstract}

Key words: Antipsychotic drugs; Off-label use; Prescription habits; Psychotic disorders; Affective disorders

Received: 21.07 .05

Accepted: 19.10 .05

\section{Introduction}

Recently there has been an increasing interest in understanding the factors that influence the prescribing of psychotropic drugs. Characteristics of the health care system, physician management style, physician specialty and training, public attitudes, drug cost and availability, patient preferences, education, marketing and formulary have been reported to have some impact on the prescription habits. ${ }^{1-5}$ On the other hand, it is reported that the patient's characteristics were taken less into consideration when physicians prescribe for adult patients. ${ }^{3,4}$

\section{Correspondence:}

Dr D Zullino

Service d'abus de substances,

Hôpitaux Universitaires de Genève,

Rue Verte 2, 1205 Genève, Switzerland

email: Daniele.Zullino@hcuge.ch
A major concern in studies on drug utilisation during the 1970s and 1980s was the appropriateness of the practices of psychotropic drug prescribing. Many articles on this topic pointed out the frequent lack of concordance between psychiatric diagnoses and the prescribed psychotropic medications $s^{6,7}$, i.e. their off label use. This may be particularly true with antipsychotic medications, due in part to their sedative properties, and thus frequently used without a need of antipsychotic effect. ${ }^{8}$

The arrival of the newer atypical antipsychotics has achieved rapid acceptance by prescribers because of enhanced safety profiles, relative to those observed with conventional antipsychotics. ${ }^{9}$ Besides their evident antipsychotic efficacy, they have been found to offer a larger pharmacological profile than conventional antipsychotic ${ }^{10}$, with some efficacy in depressive and anxious symptoms as well as suicide prevention and in mood stabilization. This has, combined with the favourable tolerance profile, led to broad off-label utilization.

Whereas several of the actual off-label utilizations are investigated with regard to their responsiveness to the newer 
antipsychotics, no conclusive data is generally available with regard to the specific prescribing parameters, such as dose, treatment duration, co-medication, etc.

The aim of the present survey is to study the prescribing habits of hospital psychiatrists with regard to antipsychotic drugs, comparing patients treated for psychoses or other registered indications with patients receiving off-label antipsychotic treatment.

\section{Methods}

The present study was undertaken as part of the AMSP project (Arzneimittelsicherheit in der Psychiatrie = drug safety in psychiatry), which is a program for continuous assessment of adverse drug reactions in psychiatric inpatients under naturalistic conditions of routine clinical treatment. The methodology has been described elsewhere. ${ }^{11-13}$ Currently, more than 35 German and Swiss sites are participating. Data on drug use in the participating hospitals are based on two reference days per year. All drugs given on a reference day are recorded along with age, sex, and diagnosis (ICD-10) for all patients under surveillance. The daily dosage is also recorded.

The data of the present study are drawn out of 5 reference days from 1999 to 2001 in the 98-bed psychiatric hospital of the University of Lausanne, Switzerland. Presently the mean hospitalisation duration is 20 days and the nurse/bed ratio is 0.95 .

\section{Definition of drug classes}

The group of atypical antipsychotics was defined as including clozapine, olanzapine, risperidone, quetiapine and amisulpride. All other antipsychotics were classed as conventional antipsychotics.

Among the conventional antipsychotics, two subclasses were identified: "sedative" and "high potency". Sedative antipsychotics were levomepromazine, promazine, clothiapine, thioridazine, and chlorprotixen. High potency antipsychotics were zuclopenthixol, haloperidol, penfluridol, flupenthixol and fluphenazine.

Benzodiazepines were classified as one group, including sedative and hypnotic drugs. As sedative benzodiazepines were also often used as hypnotics, the different indications were difficult to assess. Nonbenzodiazepine hypnotics formed a further drug class, including zolpidem, zopiclone and zaleplon. Further classified were anticonvulsants, lithium salts, anticholinergics, and somatic drugs.

Three antipsychotic drug dose ranges were defined (see Table I).

\section{Table I: Defined dose ranges for antipsychotic drugs}

\begin{tabular}{|l|l|l|l|}
\hline \multicolumn{5}{|c|}{ Dose (Mg) } \\
\hline & low & medium & high \\
\hline Levomepromazine & $<100$ & $100-200$ & $>200$ \\
risperidone & $<3$ & $3-4$ & $>4$ \\
olanzapine & $<10$ & $10-20$ & $>20$ \\
clozapine & $<200$ & $200-400$ & $>400$ \\
clothiapine & $<80$ & $80-120$ & $>120$ \\
clopenthixol & $<20$ & $20-40$ & $>40$ \\
haloperidol & $<6$ & $6-10$ & $>10$ \\
penfluridol & $<20 /$ week & $21-40$ & $>40$ \\
chlorprothixen & $<45$ & $45-90$ & $>90$ \\
flupenthixol & $<4$ & $4-6$ & $>6$ \\
quetiapine & $<300$ & $300-600$ & $>600$ \\
fluphenazine & $<20$ & $20-50$ & $>50$ \\
thioridazine & $<100$ & $100-200$ & $>200$ \\
amisulpride & $<300$ & $300-600$ & $>600$ \\
\hline
\end{tabular}

\section{Wards}

Data on prescriptions was collected from 5 different wards. Two of these wards are specialized in the treatment of patients with a diagnosis of schizophrenia spectrum disorders and are supervised by the same senior physicians. Two wards are aimed at treating anxiety and affective disorders, both again run by the same team of physicians. The fifth ward treats predominantly patients with cluster B and C personality disorders.

\section{Analyses}

In descriptive data analyses, means and standard deviations were calculated for numerical variables while frequency category values and percentages are reported for nominal variables. In exploratory analyses, the differences between groups were tested with chi-square tests (for nominal variables) and analyses of variance for numerical ones.

Three predictive models were built with multivariate logistic regression analysis. Stepwise binary logistic regression analysis was used to determine factors predicting the prescription of atypical antipsychotics and the prescription of benzodiazepines. The forward stepwise method using likelihood-ratio statistic was performed. The third model, predicting the antipsychotic dose range used was analysed by stepwise multinominal logistic regression. Multinominal logistic regression broke the regression up into a series of binary regressions comparing each group to a baseline group, which we determined to be the low dose range group.

The data were analysed using the SPSS for Windows program, version 12.0.

\section{Results \\ Characteristics of the sample}

The prescriptions of 202 patients were assessed. The mean ( \pm SD) age was $38.6 \pm 12.2$ (range $18-64$ ). The proportion of women was $43.1 \%$. There were no differences between index days with regard to age and sex distribution.

The distribution regarding their primary ICD-10 diagnosis was: Mental and behavioural disorders due to psychoactive substance use (F10): 9 (4.5\%); Schizophrenia, schizotypal and delusional disorders (F20): 122 (60.4\%); Mood disorders (F30): 39 (19.3\%); Behavioural syndromes associated with physiological disturbances and physical factors (F50): 6 (3.0\%); Disorders of adult personality and behaviour (F60): 24 (1 1.9\%).

\section{Number of prescribed drugs per patient and co-medications} The mean number of drugs administered was $4.0 \pm 1.8$ (range $1-10$ ), and the mean number of prescribed antipsychotics was $1.3 \pm 0.5$ (range $1-3$ ).

Fifty patients received nonbenzodiazepine hypnotics (24.8 \%), 117 (57.9\%) had benzodiazepines prescribed, 70 (34.7\%) antidepressants, 38 (18.8\%) anticonvulsants, 28 (13.9\%) lithium, $54(26.7 \%)$ anticholinergics, and 83 (41.1\%) somatic drugs.

\section{Atypical vs. conventional antipsychotics}

Patients treated with atypical antipsychotics $(n=67)$ were compared to those receiving conventional antipsychotics ( $n=99)$, subjects being prescribed drugs from both classes forming a third group ( $\mathrm{n}=36$ ). There was no difference with regard to age and sex between the three groups. As expected, the mean number of drugs was different between the first two groups (atypical or conventional antipsychotic) and the third group (combination): 3.6 
$\pm 1.7,3.9 \pm 1.7$ and $4.8 \pm 1.8$ respectively $(p<0.01)$ The same was true for the mean number of antipsychotics per patient: for patients with atypical antipsychotics $1.0 \pm 0.1$, for those with conventional drugs $1.3 \pm 0.5$, and for those with a combination of both $2.1 \pm 0.2(p<0.001)$. No differences were found with regard to number of comedications: atypical antipsychotics $2.6 \pm 1.7$, conventional antipsychotics $2.6 \pm 1.8$, combination $2.8 \pm 1.8$.

The proportion of patients treated concomitantly with different substance classes are shown in Table II. Patients treated with conventional antipsychotics were less likely to receive antidepressants ( $\mathrm{p}<$ 0.05), whereas patients treated with atypical antipsychotics were less likely to receive anticholinergics $(\mathrm{p}<0.01)$. Two observations of particular interest were that twelve percent of patients receiving atypical antipsychotics had concomitant treatment with anticholinergics, and patients receiving an "atypical/conventional treatment combination" presented with a similar percentage of anticholinergic treatment.

The distribution with regard to dose ranges showed significant differences between the three groups. Patients treated with atypical antipsychotics received mainly medium doses, patients treated with combination of both antipsychotic classes mostly high doses, whereas the group receiving conventional drugs was more evenly distributed $(\mathrm{p}<0.001)$.

\section{Diagnostic groups and prescription habits}

Patients were classified in 3 diagnostic groups: (i) patients with (F20) psychotic disorders, (ii) patients with manic episodes (F30, F31.1, F31.2) and depressive episodes with psychotic symptoms (F32.3, F33.3), and (iii) patients with other disorders.
Table II: Comparison of patients treated with atypical antipsychotics with those treated with conventional antipsychotics

\begin{tabular}{l|l|l|l|ll|} 
& $\begin{array}{l}\text { Atypical } \\
(n=67)\end{array}$ & $\begin{array}{l}\text { Conventional } \\
(n=99)\end{array}$ & $\begin{array}{l}\text { Both } \\
(n=36)\end{array}$ & \\
\hline Comedication (drug class) & & & & & \\
\hline Antidepressants & $40.3 \%$ & $26.3 \%$ & $47.2 \%$ & $\mathrm{Chi}^{2}(2)=6.55$ & $*$ \\
Benzodiazepines & $64.2 \%$ & $51.5 \%$ & $63.9 \%$ & $\mathrm{Chi}^{2}(2)=3.27$ & $\mathrm{~ns}$ \\
Hypnotics & $20.9 \%$ & $26.3 \%$ & $27.8 \%$ & $\mathrm{Chi}^{2}(2)=0.83$ & $\mathrm{~ns}$ \\
Anticonvulsants & $17.9 \%$ & $22.2 \%$ & $11.1 \%$ & $\mathrm{Chi}^{2}(2)=2.19$ & $\mathrm{~ns}$ \\
Lithium & $14.9 \%$ & $15.2 \%$ & $8.3 \%$ & $\mathrm{Chi}^{2}(2)=1.12$ & $\mathrm{~ns}$ \\
Anticholinergics & $11.9 \%$ & $34.3 \%$ & $33.3 \%$ & $\mathrm{Chi}^{2}(2)=11.21$ & $* *$ \\
Somatic drugs & $43.3 \%$ & $38.4 \%$ & $44.4 \%$ & $\mathrm{Chi}^{2}(2)=0.60$ & $\mathrm{~ns}$ \\
& & & & & \\
Antipsychotic dose received & & & & & \\
Dose range & $16.4 \%$ & $22.2 \%$ & $2.8 \%$ & $\mathrm{Chi}^{2}(4)=60.92$ & $* * *$ \\
$\quad$ Low & $71.6 \%$ & $42.4 \%$ & $11.1 \%$ & & \\
$\quad \begin{array}{l}\text { Medium } \\
\text { High }\end{array}$ & $11.9 \%$ & $35.4 \%$ & $86.1 \%$ & & \\
\hline
\end{tabular}

Group (1) and (2) formed the class of patients receiving an antipsychotic for a registered indication, and the prescriptions in group (3) can be considered as off-label.

The 3 groups differed with regard to age $[F(2)=6.12$; $\mathrm{p}=0.003]$ : psychotic patients $37.7 \pm 11.8$ years, patients with affective disorders $44.4 \pm 13.9$ years, patients with off-label indications $35.7 \pm 9.9$ years. The differences between patients with a registered indication vs. patients with off-label indications was significant $[t(199)=2.45 ; p=0.015]$, whereas the difference between psychotic patients vs. the other two diagnostic categories was not $[F(199)=-1.38 ; p=0.169]$.

As shown in Table III, the diagnostic groups differed with regard to number of prescribed drug per patient $(\mathrm{p}<0.01)$, number of prescribed antipsychotic per patient $(p<0.001)$ and number of prescribed comedication drugs $(p<0.001)$. Contrasting registered indications (groups 1 and 2 ) with off-

\begin{tabular}{|c|c|c|c|c|c|c|c|c|c|c|c|c|}
\hline & \multirow[t]{2}{*}{$\begin{array}{l}\text { Psychosi } \\
(n=80)\end{array}$} & & \multirow{2}{*}{\multicolumn{2}{|c|}{$\begin{array}{l}\text { Labelled affective } \\
\text { disorder } \\
(n=122)\end{array}$}} & \multirow{2}{*}{\multicolumn{2}{|c|}{$\begin{array}{l}\text { Off label } \\
\text { indication } \\
(n=51)\end{array}$}} & & \multirow[b]{2}{*}{$p$} & \multicolumn{2}{|c|}{$\begin{array}{l}\text { Registered indications } \\
\text { vs off-label use }\end{array}$} & \multicolumn{2}{|c|}{$\begin{array}{l}\text { Psychosis } \\
\text { vs others }\end{array}$} \\
\hline & & & & & & & & & $t$ & $p$ & $t$ & $p$ \\
\hline $\begin{array}{l}\text { Number drugs } \\
\text { Number Antipsychotics } \\
\text { Number Comedication }\end{array}$ & $\begin{array}{l}\text { mean } \\
3.54 \\
1.43 \\
2.11\end{array}$ & $\begin{array}{l}S D \\
1.61 \\
0.56 \\
1.53\end{array}$ & $\begin{array}{l}\text { mean } \\
4.79 \\
1.05 \\
3.74\end{array}$ & $\begin{array}{l}S D \\
1.64 \\
0.32 \\
1.63\end{array}$ & $\begin{array}{l}\text { mean } \\
4.39 \\
1.22 \\
3.17\end{array}$ & $\begin{array}{l}\mathrm{SD} \\
1.95 \\
0.47 \\
1.82\end{array}$ & $\begin{array}{l}F(2)=9.84 \\
F(2)=9.01 \\
F(2)=17.89\end{array}$ & $\begin{array}{l}0.003 \\
<0.001 \\
<0.001\end{array}$ & $\begin{array}{l}t(199)=-0.726 \\
t(199)=0.210 \\
t(199)=-0.828\end{array}$ & $\begin{array}{l}0.469 \\
0.834 \\
0.409\end{array}$ & $\begin{array}{l}t(199)=-4.324 \\
t(199)=-4.002 \\
t(199)=-5.706\end{array}$ & $\begin{array}{l}<0.001 \\
<0.001 \\
<0.001\end{array}$ \\
\hline $\begin{array}{l}\text { Comedication (drug class) } \\
\text { Antidepressants } \\
\text { Benzodiazepines } \\
\text { Hypnotics } \\
\text { Anticonvulsants } \\
\text { Lithium } \\
\text { Anticholinergics } \\
\text { Somatic drugs }\end{array}$ & \begin{tabular}{l|}
27.9 \\
54.9 \\
18.0 \\
11.5 \\
7.4 \\
30.3 \\
36.1
\end{tabular} & $\begin{array}{l}\% \\
\% \\
\% \\
\% \\
\% \\
\% \\
\\
\%\end{array}$ & $\begin{array}{l}41.0 \\
64.1 \\
38.5 \\
41.0 \\
41.0 \\
30.8 \\
51.3\end{array}$ & $\begin{array}{l}\% \\
\% \\
\% \\
\% \\
\% \\
\% \\
\% \\
\end{array}$ & $\begin{array}{l}48.8 \\
61.0 \\
31.7 \\
19.5 \\
7.3 \\
12.2 \\
46.3\end{array}$ & $\begin{array}{l}\% \\
\% \\
\% \\
\% \\
\% \\
\% \\
\% \\
\%\end{array}$ & $\begin{array}{l}\operatorname{Chi}^{2}(2)=6.79 \\
\operatorname{Chi}^{2}(2)=1.22 \\
\operatorname{Chi}^{2}(2)=7.96 \\
\operatorname{Chi}^{2}(2)=16.91 \\
\operatorname{Chi}^{2}(2)=29.87 \\
\operatorname{Chi}^{2}(2)=5.55 \\
\operatorname{Chi}^{2}(2)=3.41\end{array}$ & $\begin{array}{l}0.034 \\
0.543 \\
0.019 \\
<0.001 \\
<0.001 \\
0.062 \\
0.182\end{array}$ & & & & \\
\hline $\begin{array}{l}\text { Class of antipsychotics } \\
\text { Atypical } \\
\text { Conventional } \\
\text { Both }\end{array}$ & $\begin{array}{l}35.2 \\
41.8 \\
23.0\end{array}$ & $\begin{array}{l}\% \\
\% \\
\%\end{array}$ & $\begin{array}{l}35.9 \\
61.5 \\
2.6\end{array}$ & $\begin{array}{l}\% \\
\% \\
\%\end{array}$ & $\begin{array}{l}24.4 \\
58.5 \\
17.1\end{array}$ & $\begin{array}{l}\% \\
\% \\
\%\end{array}$ & $\operatorname{Chi}^{2}(4)=11.41$ & 0.022 & & & & \\
\hline $\begin{array}{l}\text { Proportion hi-potency } \\
\text { (N Conventionals = 133) } \\
\text { Proportion low-potency } \\
\text { (N Conventionals = 133) }\end{array}$ & $\begin{array}{l}65.4 \\
56.4\end{array}$ & $\begin{array}{l}\% \\
\%\end{array}$ & $\begin{array}{l}72.0 \\
32.0\end{array}$ & $\begin{array}{l}\% \\
\%\end{array}$ & $\begin{array}{l}20.0 \\
83.3\end{array}$ & $\begin{array}{l}\% \\
\%\end{array}$ & $\begin{array}{l}\operatorname{Chi}^{2}(2)=21.20 \\
\operatorname{Chi}^{2}(2)=14.91\end{array}$ & $\begin{array}{l}<0.001 \\
<0.001\end{array}$ & & & & \\
\hline $\begin{array}{l}\text { Dose range } \\
\text { Low } \\
\text { Medium } \\
\text { High }\end{array}$ & $\begin{array}{l}9.2 \\
28.9 \\
61.8\end{array}$ & $\begin{array}{l}\% \\
\% \\
\%\end{array}$ & $\begin{array}{l}28.0 \\
48.0 \\
24.0\end{array}$ & $\begin{array}{l}\% \\
\% \\
\%\end{array}$ & $\begin{array}{l}30.0 \\
30.0 \\
40.0\end{array}$ & $\begin{array}{l}\% \\
\% \\
\%\end{array}$ & $\operatorname{Chi}^{2}(4)=15.54$ & 0.004 & & & & \\
\hline
\end{tabular}


label indications (group 3) revealed no significant differences with regard to these observations. Contrasting psychotic patients with the two other groups revealed significant differences for all 3 comparisons $(p<0.001)$.

Several differences appeared between the diagnostic groups with regard to comedications: fewer psychotic patients received antidepressant $(\mathrm{p}<0.05)$ and nonbenzodiazepine hypnotics $(p<0.001)$ than patients from the other two groups. More patients from the affective disorders group received anticonvulsants than subjects of the two comparative groups $(\mathrm{p}<0.001)$.

Patients with affective disorders rarely received a combination of an atypical and a conventional antipsychotic, whereas patients with off-label indications received atypical antipsychotics less often than the two comparison groups $(\mathrm{p}<0.05)$.

\section{Secular effects}

Only one significant change over the 5 index days was observed. The percentage of patients treated with atypical antipsychotics increased over the observation period. The proportions for the 5 index days were 25.5\%, 22.5\%, 29.4\%, $33.3 \%$ and $53.3 \%$ respectively, the differences being statistically significant $(p<0.05)$.

As shown in Figure 1, there was a secular trend for patients without psychosis, who were treated less often with atypical antipsychotics than patients with the diagnosis of a psychosis at the beginning of the observation with the difference disappearing by the last observation.

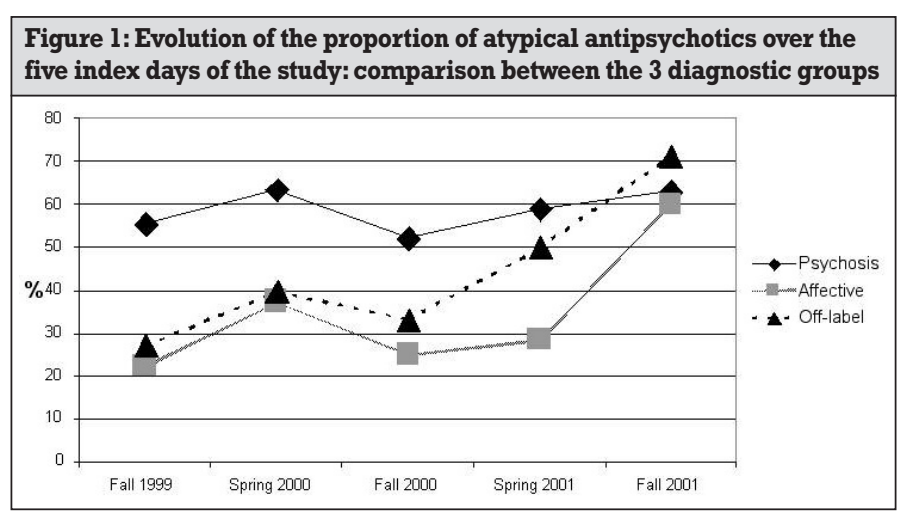

\section{Logistic regressions}

Three predictive models were built with multivariate logistic regression analysis.

A first model was computed to determine factors predicting the prescription of atypical antipsychotics (Table IV). The following parameters were entered into the stepwise

\begin{tabular}{|c|c|c|c|}
\hline \multicolumn{4}{|c|}{$\begin{array}{l}\text { Table IV: Stepwise logistic regression model for use of atypical } \\
\text { antipsychotics }\end{array}$} \\
\hline & \multicolumn{3}{|c|}{ Atypical antipsychotics } \\
\hline & $O R^{a}$ & $p$ & $C I$ \\
\hline \multicolumn{4}{|l|}{ Diagnostic class } \\
\hline Psychosis & 1 & 0.007 & \\
\hline $\begin{array}{l}\text { Registered affective disorder } \\
\text { Off-label use }\end{array}$ & 0.38 & 0.010 & $0.18-0.79$ \\
\hline UIse of an antidenrescant & 273 & נחم00 & $151-492$ \\
\hline & & & \\
\hline
\end{tabular}

Table V: Stepwise logistic regression model for use of benzodiazepines

\begin{tabular}{|l|c|c|c|}
\hline & \multicolumn{3}{|c|}{ Benzodiazepines } \\
& $O^{a}$ & $p$ & \\
\hline Use of an antidepressant & 2.50 & 0.002 & $1.40-4.44$ \\
Use of a nonbenzodiazepine hypnotic & 1.97 & 0.049 & $1.00-3.85$ \\
\hline \multicolumn{4}{|l|}{ 'Odds ratio for the probability of receiving a benzodiazepine } \\
\hline
\end{tabular}

logistic regression model: index day, sex, age, diagnostic class, and use of nonbenzodiazepine hypnotics,

benzodiazepines, antidepressants, anticonvulsants, lithium and somatic drugs. The parameters "diagnostic class" and "prescription of an antidepressant" were retained. The positive predictive value was $57.8 \%$.

The second model was aimed to determine factors predicting the prescription of concomitant benzodiazepines (Table V). The parameters entered into the stepwise logistic regression model were: index day, sex, age, diagnostic class, and use of an atypical antipsychotic, nonbenzodiazepine hypnotics, antidepressants, anticonvulsants, lithium and somatic drugs. The parameters retained were the "use of antidepressants" and the "use of nonbenzodiazepine hypnotics". The positive predictive value was $62.3 \%$.

Stepwise multinominal logistic regression analysis was used to assess factors associated with the use of medium range and high range doses (Table VI). The group of individuals having received the antipsychotic medication at a low dose range were defined as the reference group. The following parameters were entered into the model: age, index day, sex, diagnostic class, use of nonbenzodiazepine hypnotics, benzodiazepines, antidepressants, anticonvulsants, lithium, antiparkinsonians, and somatic drugs. The parameters of the "diagnostic class" and the "prescription of antiparkinsonian drugs" were retained. The positive predictive value was $50.5 \%$.

\section{Table VI: Logistic regression model for choice of antipsychotic dose range}

\begin{tabular}{|c|c|c|c|c|c|c|}
\hline & \multicolumn{3}{|c|}{ Medium doses } & \multicolumn{3}{|c|}{ High doses } \\
\hline & $O R$ & $p$ & $C I$ & $O R$ & $p$ & $C I$ \\
\hline $\begin{array}{l}\text { Diagnostic class } \\
\text { Psychotic } \\
\text { Registered affective disorder } \\
\text { Off-label indication }\end{array}$ & $\begin{array}{l}10.11 \\
4.75 \\
3.94\end{array}$ & $\begin{array}{l}0.001 \\
0.020 \\
0.068\end{array}$ & $\begin{array}{l}2.69-38.08 \\
1.27-17.69 \\
0.91-17.10\end{array}$ & $\begin{array}{l}20.66 \\
2.40 \\
7.77\end{array}$ & $\begin{array}{c}<0.001 \\
0.239 \\
0.007\end{array}$ & $\begin{array}{l}5.49-77.84 \\
0.56-10.27 \\
1.74-34.69\end{array}$ \\
\hline Antiparkinsonian & 2.89 & 0.113 & $0.78-10.72$ & 8.29 & 0.002 & $2.18-31.46$ \\
\hline
\end{tabular}


Presenting with a primary diagnosis of a psychotic disorder was associated with a 10-fold risk of having received the antipsychotic medication in the middle dosage range and not in the low dosage range $(\mathrm{p}<0.001)$, and a 20 -fold risk of receiving antipsychotics in the high dose range $(p<0.001)$. For patients with an affective disorder considered a registered indication for an antipsychotic treatment, the odds ratio of receiving a middle dose treatment was $4.75(p<0.05)$, but the increased relative risk to receive a high dose instead of a lowdose was not significant. Whereas patients treated on an offlabel basis did not have a significantly higher risk to receive a middle dose compared to a low dose regimen, their odds ratio of receiving their antipsychotics in high doses was 7.77 higher than for low doses $(p<0.007)$. When patients received an antiparkinsonian, the risk that they also were treated with high antipsychotic doses was increased 8.29-fold compared to low doses $(\mathrm{p}<0.01)$.

\section{Discussion}

Like in previous survey studies in psychiatric hospitals ${ }^{14-18}$, polypharmacy was frequent in our sample, the mean number of prescribed drugs being 4 and ranging from 1 to 10 drugs per patient. Whereas polypharmacy has often been considered as malpractice in earlier studies ${ }^{19-22}$, it has become increasingly apparent nowadays, that psychiatric polypharmacy can have some advantages, i.e. to further improve sleep, have a more potent anxiolytic or sedative effect and to overcome treatment resistance. ${ }^{17,23,24}$ Such considerations may also have played a role for the prescription habits of the physicians in our study, as 58\% of the patients in our sample received benzodiazepines concomitantly to the antipsychotic, and $25 \%$ a nonbenzodiazepine hypnotic. This seemed to be particularly true for patients with affective or other disorders, as psychotic patients received fewer co-medications in general and especially fewer antidepressant and nonbenzodiazepine hypnotics.

Interestingly, conventional antipsychotics were more often associated with antidepressant co-medication. This may be due to differences between the two diagnostic classes with regard to secular trend.s. The use of newly introduced atypical antipsychotic drugs spread rapidly in the treatment of psychoses (the primary indication), with a certain latency in pharmacotherapy of affective disorders (mania and psychotic depression) and off-label indications.

Whereas patients with the diagnosis of a psychosis were already treated in more than $50 \%$ of the cases with an atypical agent at the beginning of the observation period, the proportion was $25 \%$ in patients without psychosis. The difference disappeared over the five index days with the proportion of prescribed atypical antipsychotics being almost $65 \%$ in all diagnostic groups during the last index day. The most convincing hypothesis to explain these observations would be that the newer drugs were used with less hesitation firstly in approved indications, and that prescribing physicians used newer drugs off-label only after having accumulated some experience in approved indications. This effect may even have been reinforced by the fact that our hospital wards are organized according to diagnostic groups (schizophrenia, affective disorders, personality disorders, triage unit). Therefore physicians working in the units with a high prevalence of psychotic patients had accumulated experiences with newer drugs more rapidly. One can furthermore hypothesize that due to the usual turn-over of trainees the prescribing habits developed on the specialised wards was subsequently "exported" to the whole hospital.

Further interesting results are the dose differences between patients treated for an approved indication compared to off-label use. Whereas the proportion of patients being treated with antipsychotics at medium doses was similar, high doses were more frequent in patients with approved indications, low doses were more frequent for off-label use. Once again, physicians treating patients with approved indications seem to be less hesitant when using antipsychotics.

The observations made with regard to co-medications confirmed what was expected. Antidepressants, nonbenzodiazepine hypnotics and mood stabilizers were more often given to patients without psychosis, most likely in order to treat their primary disease, using antipsychotics probably most often as sedatives.

The use of atypical antipsychotics itself seems to be associated with some particular prescribing habits. As could be expected, the use of anticholinergics was lower. Atypical drugs were particularly used in medium doses, whereas monotherapy with conventional drugs were in more than one third of the cases prescribed in higher doses. This last observation is difficult to interpret. One highly speculative hypothesis could be that the prescribing physicians were more confident in the effects of atypical antipsychotics, using them less often in high doses.

The results of this study need to be viewed against their methodological limitations. The data are based on five index day surveys, i.e. five crossover data samples. The secular trends found in this study should therefore be interpreted with particular caution. Furthermore, the measured data do not always reflect the intended medication for one given patient, which is a more dynamic process. This will be particularly the case in patients having been hospitalised only recently, whose medication is possibly not yet stabilized. The diagnoses were derived from the medical records ahd could therefore not be considered as valid as diagnoses which would have been determined by structured interviews.

\section{Conclusion}

Whereas previous studies have stressed, that prescribing habits are primarily influenced by doctors' characteristics and contextual factors ${ }^{1-5}$ and less likely by patient's characteristics $^{3,4}$, our study suggests that, at least shortly after the introduction of newer therapeutic agents, patient's diagnosis may influence drug choice, dose and comedications. While no analogous data on off label prescribing has been published, to our knowledge, in Africa, it is possible that similar observations may be made in the South African context.

\section{References}

1. Olfson M, Pincus HA, Dial TH. Professional practice patterns of U.S. psychiatrists. Am J Psychiatry 1994;151(1):89 - 95.

2. Tylee A. Depression in the community: physician and patient perspective.J Clin Psychiatry 1999;60(suppl 7):12 - 16.

3. Egberts ACG, Veenstra M, de Jong-van den Berg LTW. Antidepressant 
drug choice for first users in two regions in the Netherlands. Pharm. World Sci. 1999;21 (3):132 - 136.

4. Linden $M$, Lecrubier Y, Bellantuono C, Benkert O, Kisely S, Simon G. The prescribing of psychotropic drugs by primary care physicians: an international collaborative study. J Clin Psychopharmacology 1999;19(2):132 - 140 .

5. Laux G, Baier D. Quality-monitoring of psychotropic drug therapy in post-marketing surveillance. Pharmacopsychiatry 1997;30(suppl):21 27.

6. Tinsley JA, Shadid GE, Li H, Offord KP, Agerter DC. Psychotropic prescribing practices and educational needs: a survey of family physicians and psychiatrists. Gen Hosp Psychiat. 1998;20:360 - 367

7. Bouhassira M, Allicar MP, Blachier C, Nouveau A, Rouillon F. Which patients receive antidepressants? A 'real world' telephone study.J Affective Disorders 1998:49:19 - 26.

8. Müller WE. Pharmakologische Grundlagen der Therapie mit Neuroleptika ausserhalb ihrer Anwendung bei Schizophrenen. In. Berlin: Springer; 1999. p. 15 - 30

9. Pincus HA, Tanielian TL, Marcus SC, Olfson M, Zarin DA, Thompson J, et al. Prescribing trend in psychotropic medications: primary care, psychiatry, and other medical specialities. JFMA 1998;279:526 - 531.

10. Buckley PF. Broad therapeutic uses of atypical antipsychotic medications. Biological Psychiatry 2001;50(11):912 - 924.

11. Grohmann R, Rüther E, Engel RR, Hippius H. Assessment of adverse drug reactions in psychiatric inpatients with the AMSP drug safety program: methods and first results for tricyclic antidepressants and SSRI. Pharmacopsychiatry 1999;32:21 - 28

12. Zullino D, Baumann P, Grohmann R, Greil W. L'importance de la pharmacovigilance en psychiatrie - Le Projet AMSP (Arzneimittelsicherheit in der Psychiatrie). Revue médicale de la Suisse Romande 2000;120:105 - 109.

13. Zullino DF, Horvath A, Greil W, Grohmann R, Hippius H, Eich P, et al. Die Bedeutung der Pharmakovigilanz in der Psychiatrie: das AMSPProjekt (Arzneimittelsicherheit in der Psychiatrie). Schw Arch Neurol
Psychiatr 2002;153:266 - 271.

14. Muijen M, Silverstone T. A comparative hospital survey of psychotropic drug prescribing. BrJ Psychiatry 1987;150:501 - 504.

15. Diamond H, Tislow R, Snyder T, Rickels K. Peer review of prescribing patterns in a CMHC. Am J Psychiatry 1976;133:697 - 699.

16. Lippert E, Aigner JM, Grohmann R, Klein HE, Schmauss M, Rüther E. Anwendungshäufigkeiten und Dosierungen von Psychopharmaka an psychiatrischen Versorgungskrankenhäusern - Ergebnisse aus dem Arzneimittelüberwachungsprojekt Bayern. Psychopharmakotherapie 1996:3:178-183.

17. Rittmansberger H, Meise U, Schauflinger K, Horvath E, Donat $H$, Hinterhuber H. Polypharmacy in psychiatric treatment. Patterns of psychotropic drug use in Austrian psychiatric clinics. Eur Psychiatry 1999;14:33 - 40.

18. Ambühl B, Würmle O, Michel G. Die Verschreibungspraxis von Psychopharmaka in einer psychiatrischen Unversitätsklinik. Psychiatr Prax. 1993;20:70 - 73.

19. Pincus HA, Zarin DA, Tanielian TL, Johnson JL, West JC, Pettit AR, et al. Psychiatric patients and treatments in 1997. Arch Gen Psychiatry 1999;56:441 - 449

20. Clark AF, Holden NL. The persistence of prescribing habits: a survey and follow-up of prescribing to chronic hospital in-patients. Br J Psychiatry 1987;150:88 - 91.

21. Michel K, Kolakowska T. A survey of prescribing psychotropic drugs in two psychiatric hospitals. Br J Psychiatry 1981;138:217 - 221.

22. Nichol MB, Stimmel GL, Lange SC. Factors predicting the use of multiple psychotropic medications. J Clin Psychiatry 1995;56(2):60 66

23. Zullino D, Baumann P. Lithium augmentation in depressive patients not responding to selective serotonin reuptake inhibitors. Pharmacopsychiatry 2001;34:1 - 9.

24. Zullino D, Bondolfi G, Baumann P. The serotonin paradox: negative symptoms and SSRI augmentation. Int J Psych Clin Prac. 1998;2:19 26. 